Fixed Point Theory, 23(2022), No. 1, 311-330

DOI: $10.24193 /$ fpt-ro.2022.1.20

http://www.math.ubbcluj.ro/ nodeacj/sfptcj.html

\title{
LINEAR AND SUPERLINEAR CONVERGENCE OF AN INEXACT ALGORITHM WITH PROXIMAL DISTANCES FOR VARIATIONAL INEQUALITY PROBLEMS
}

\author{
E.A. PAPA QUIROZ* AND S. CRUZADO ACUÑA** \\ *Universidad Nacional Mayor de San Marcos and Universidad Privada del Norte \\ E-mail: erikpapa@gmail.com \\ ${ }^{* *}$ Universidad Nacional Mayor de San Marcos \\ E-mal: scruzadoa@gmail.com
}

\begin{abstract}
This paper introduces an inexact proximal point algorithm using proximal distances with linear and superlinear rate of convergence for solving variational inequality problems when the mapping is pseudomonotone or quasimonotone. This algorithm is new even for the monotone case and from the theoretical point of view the error criteria used improves recent works in the literature. Key Words and Phrases: Variational inequalities, proximal distances, proximal point algorithms, quasimonotone and pseudomonotone mappings.
\end{abstract}

2020 Mathematics Subject Classification: 90C33, 47H10.

\section{REFERENCES}

[1] A. Auslender, M. Teboulle, Interior projection-like methods for monotone variational inequalities, Math. Program, Serie A, (2005), no. 104, 39-68.

[2] A. Auslender, M. Teboulle, Interior gradient and proximal methods for convex and conic optimization, SIAM Journal of Optimization, 16(2006), no. 3, 697-725.

[3] N. Baygorrea, E.A. Papa Quiroz, N. Maculan, Inexact proximal point methods for quasiconvex minimization on Hadamard manifolds, J. Oper. Res. Soc. China, 4(2016), no. 4, 397-424.

[4] A. Bnouhachem, X. Qin, An inertial proximal Peaceman-Rachford splitting method with SQP regularization for convex programming, J. Nonlinear Funct. Anal., 50(2020), 1-17.

[5] S.A. Brito, J.X. da Cruz Neto, J.O. Lopes, P.R. Oliveira, Interior algorithm for quasiconvex programming problems and variational inequalities with linear constraints, J. Optim Theory Appl., 154(2012), no. 1, 217-234.

[6] X. Cai, A proximal point algorithm with asymmetric linear term, Optimization Letters, 13(2019), no. 4, 777-793.

[7] L.C. Ceng, Two inertial linesearch extragradient algorithms for the bilevel split pseudomonotone variational inequality with constraints, J. Appl. Numer. Optim., 2(2020), 213-233.

[8] G. Chen, M. Teboulle, Convergence analysis of a proximal-like minimization algorithm using Bregman functions, SIAM J. Optim., (1993), no. 3, 538-543.

[9] J. Eckstein, Approximate iterations in Bregman-function-based proximal algorithms, Mathematical Programming, 83(1998), 113-123.

[10] J. Eckstein, D.P. Bertsekas, On the Douglas-Rachford splitting method and the proximal algorithm for maximal monotone operators, Mathematical Programming, 55(1992), no. 3, 293-318. 
[11] F. Facchinei, J.S. Pang, Finite-Dimensional Variational Inequalities and Complementarity Problems, Vol. I and II, Springer Series in Operations Research, Springer-Verlag, New York, 2003.

[12] A. Gibali, A new Bregman projection method for solving variational inequalities in Hilbert, Pure Appl. Funct. Anal., 3(2018), 403-415.

[13] D. Han, A generalized proximal-point-based prediction-correction method for variational inequality problems, Journal of Computational and Applied Mathematics, 221(2008), 183-193.

[14] P.T. Harker, J.S. Pang, Finite-dimensional variational inequality and nonlinear complementarity problems: a survey of theory, algorithms and applications, Mathematical Programming, 48(1990), 161-220.

[15] D.V. Hieu, P. Cholambjiak, Modified extragradient method with Bregman distance for variational inequalities, Applicable Analysis, doi.org/10.1080/00036811.2020.1757078

[16] A. Kaplan, R. Tichatschke, Interior proximal method for variational inequalities on nonpolyhedral sets, Discuss. Math. Diff. Inclusions Control Optim., 30(2007), 51-59.

[17] A. Kaplan, R. Tichatschke, Note on the paper: interior proximal method for variational inequalities on non-polyhedral sets, Discuss. Math. Diff. Inclusions Control Optim., 30(2010), 51-59.

[18] K.C. Kiwiel, Proximal minimization methods with generalized Bregman functions, SIAM J. Control Optim., 35(1997), 1142-1168.

[19] N. Langenberg, Pseudomnonotone operators and the Bregman proximal point algorithm, J. Glob Optim., 47(2010), 537-555.

[20] N. Langenberg, An interior proximal method for a class of quasimonotone variational inequalities, J. Optim Theory Appl., 155(2012), 902-922.

[21] N. Langenberg, R. Tichatschke, Interior proximal methods for quasiconvex optimization, J. Global Optim., 52(2012), 641-661.

[22] Y. Liu, D. Sun, K. Toh, An implementable proximal point algorithmic framework for nuclear norm minimization, Mathematical Programming, 133(2012), no. 1-2, 399-436.

[23] E.A. Papa Quiroz, L. Mallma Ramirez, P.R. Oliveira, An inexact proximal method for quasiconvex minimization, European J. Operational Research, 246(2015), 721-729.

[24] E.A. Papa Quiroz, L. Mallma Ramirez, P.R. Oliveira, An inexact algorithm with proximal distances for variational inequalities, RAIRO Oper. Res., 52(2018), no. 1, 159-176.

[25] E.A. Papa Quiroz, P.R. Oliveira, An extension of proximal methods for quasiconvex minimization on the nonnegative orthant, European J. Operational Research, 216(2012), 26-32.

[26] B.T. Polyak, Introduction to Optimization, Optimization Software, New York, 1987.

[27] R.T. Rockafellar, Monotone operations and the proximal point method, SIAM J. Control Optimization, 14(1976), 877-898.

[28] R.T Rockafellar, R. Wets, Variational Analysis, Grundlehren der Mathematischen, Wissenschaften 317, Springer 1998.

[29] O. Sarmiento, E.A. Papa Quiroz, P.R. Oliveira, A proximal multiplier method for separable convex minimization, Optimization, 65(2016), no. 2, 501-537.

[30] O. Sarmiento, E.A. Papa Quiroz, P.R. Oliveira, Convergence rate of a proximal multiplier algorithm for separable convex minimization, Optimization, 66(2017), no. 2, 251-270.

[31] K. Shimizu, K. Hishinuma, H. Iiduka, Parallel computing proximal method for non-smooth convex optimization with fixed point constraints of quasi-nonexpansive mappings, Appl. SetValued Anal. Optim., (2020), no. 2, 1-17.

[32] P.J.S. Silva, J. Eckstein, Double-regularization proximal methods, with complementarity applications, Comput. Optim. Appl., 33(2006), 115-156.

[33] M.V. Solodov, Convergence rate analysis of iteractive algorithms for solving variational inequality problems, Mathematical Programming, Ser. A., 96(2003), 513-528.

[34] M.V. Solodov, B.F. Svaiter, An inexact hybrid generalized proximal point algorithm and some new results on the theory of Bregman functions, Mathematics of Operations Research, 25(2000), 214-230.

[35] G.J. Tang, N.J. Huang, An inexact proximal point algorithm for maximal monotone vector fields on Hadamard manifolds, Operations Research Letters, 41(2013), no. 6, 586-591. 
[36] M. Teboulle, Convergence of proximal-like algorithms, SIAM J. Optimization, 7(1997), no. 1, 1069-1083.

[37] P. Tseng, On linear convergence of iterative methods for the variational inequality problem, Journal of Computational and Applied Mathematics, 60(1995), 237-252.

[38] X. Wang, X. Ou, T. Zhang, J. Chen, An alternate minimization method beyond positive definite proximal regularization: convergence and complexity, J. Nonlinear Var. Anal., (2019), no. 3, 333-355.

Received: September 6, 2020; Accepted: March 22, 2021. 